\title{
Science, Mechanisms and Evidence
}

\author{
Ignacio Gimenez* \\ Universidad Nacional de Tres de Febrero (UNTREF), Buenos Aires, Argentina \\ *Corresponding author: Ignacio Gimenez, Universidad Nacional de Tres de Febrero (UNTREF), Buenos Aires, Argentina
}

\begin{tabular}{|c|c|}
\hline ARTICLE INFO & ABSTRACT \\
\hline Received: & Citation: Ignacio Gimenez. Science, Mechanisms and Evidence. Biomed J Sci \& Tech Res \\
\hline Published: 櫘 January 30, 2020 & 25(2)-2020. BJSTR. MS.ID.004163. \\
\hline
\end{tabular}

\section{Introduction}

A good way to explain what we observe is through the description of the mechanisms that generate it. This perspective, generally called mechanistic philosophy or "mechanicism", provides tools to arrive at explanations of phenomena of a very diverse nature, from protein synthesis to social processes. The very concept of mechanism can be analyzed in terms of its constituent entities and activities. One of the best-known definitions of mechanism within this philosophical framework is the following: a mechanism is entities and activities organized in such a way that they produce regular changes from initial conditions to final conditions [1]. In this sense, the way in which a mechanism is described gives an idea of how the final observed state of a phenomenon is reached from certain initial conditions. This explanatory mode has had a notorious development and is now very common in many areas of contemporary science, especially in the occasionally called "special sciences", such as biology, neuroscience and psychology.

\section{Mechanisms in Neuroscience}

In the particular case of neuroscience, Craver [2] has developed a whole conceptual framework based on mechanicism to defend his position on how neuroscientists manage to explain the phenomena they study more or less correctly. For example, action potentials are explained by ionic flows, some forms of neurotransmitter release are explained by calcium concentrations in the terminal axon of neurons, protein sequences are explained (in part) by DNA sequences. These are good explanations that most scientists and philosophers would agree upon. But bad explanations can also be found, such as that memory is explained by the vibration of vital fluids through the ventricles of the brain, that the shape of a person's skull explains his or her artistic talents, and that memory loss explains the deposition of beta amyloid in the cerebral cortex
[3]. This is why to get a good criterion of which are good and bad explanations in science (especially those of relatively recent development) is of great importance to handle explanations of the mechanisms underlying the phenomena under study. Furthermore, arriving at a good mechanistic explanation in neuroscience allows the possibility of intervening in some way in the functioning of the brain with the aim of relieving the symptoms of a neurological disease or improving some cognitive capacity. Far from being in the sphere of the merely descriptive, this brings with it practical consequences that have placed the knowledge of the component parts and activities of the mechanisms in a predominant place in the neurosciences and in the biomedical sciences.

\section{Seeing Medicine Through Mechanisms: The Case of Evidence-Based Medicine}

Another particularly interesting application of the mechanistic framework is that of evidence-based medicine. According to David Sackett [4], evidence-based medicine consists of the conscious, explicit and judicious use of the best current evidence in making decisions about the care of individual patients. The practice of evidence-based medicine involves integrating individual clinical experience with the best available external clinical evidence derived from systematic research. In this process of making decisions about patient care, it is necessary to establish the most likely causes of the observed symptoms (the diagnosis) and to determine which intervention is best suited to alleviate the diagnosed cause (the treatment). However, within the practice of evidence-based medicine, when talking about "the best available external clinical evidence" there is no one type of evidence that is completely established. There are currently what are known as evidence hierarchies, in which the order of reliability of the different types 
of evidence available for public health decision-making is made explicit [5].

For example, the one issued by the National Institute for Health and Clinical Excellence [6] in the United Kingdom. In general, these hierarchical orders of evidence that are commonly used in the practice of medicine prioritize those obtained from clinical, experimental or observational trials, and meta-analyses of large numbers of clinical studies. However, these types of studies are based on correlations between variables, i.e. they do not imply causality. For this reason, their results should be taken with caution when deciding on an appropriate treatment. With the aim of improving evidence-based medicine, Clarke and collaborators express the need to place the evidence of mechanisms on the same level as correlation studies when deciding on an appropriate treatment [7]. It should be noted that in this context, evidence of mechanisms can take many forms, from laboratory experiments, basic science literature reviews, individual patient cases, textbook consensus, to expert testimony. But how can knowledge of the mechanism of a treatment or disorder contribute to better practice of evidence-based medicine?

\section{Using Mechanisms to Improve Clinical Trials Design}

Firstly, a better understanding of the mechanism underlying a medical treatment can lead to a more appropriate design and interpretation of a clinical trial. For example, in cases where a disease may occur by different possible mechanisms, if the pathways in which a drug acts to relieve its symptoms are known, patients may be recruited in whom a diagnosis has been made based on the production of those symptoms and not others. This may also lead to deciding which variables to measure in each clinical trial [7]. In this sense, the way in which a disease occurs is reminiscent of multiple realizability phenomena [8]. An example can be found in the use of the monoclonal antibody Eculizumab to treat Hemolytic Uremic Syndrome (HUS), a serious failure in renal function. This condition can originate in several ways, one of which is from a mutation in a particular metabolic pathway ("the complement pathway") or from a toxin produced by the bacterium Shigella dysenteriae. Eculizumab is only efficient in treating HUS produced by the mutation in the complement pathway, since it has a mechanism of action that allows it to bind to one of the molecules that are defective, preventing its cleavage and thus restoring the glomerular filtrate [9]. This is why positive results are expected from the use of Eculizumab in a clinical trial in HUS patients with a genetic origin and not because of "Shigga toxin". Also, knowing the pharmacokinetic mechanisms of the drug allows us to better decide which clinical parameters to measure and at what times.

\section{Mechanisms as an External Source of Clinical Evidence}

Another aspect in which the consideration of the mechanism can be complemented with evidence from clinical studies is by acting as an external source of validation of the latter, which allows its generalizability to other populations or individuals falling outside the studied domain. This action of generalizing is based on the assumption of what is known as "universal biological response", that is, that different individuals will respond to a treatment or a drug in the same way. What happens is that many times the results of a clinical trial cannot be directly transposed to other populations with different characteristics or to other environmental conditions, since the causal mechanisms change. Generally, the assumption of generalizability works when the mechanism of action of an intervention is relatively simple and direct (e.g., individual-level studies of vaccines and dietary supplements). But this assumption is not appropriate in cases where the mechanism consists of a long and complex causal chain that may be affected in several places by the demographic characteristics of the population, the health system or the environment [10].

In this way, knowing the underlying mechanism can validate externally that the effects measured are indeed due to the causes attributed to the treatment. This aspect can be well visualized in cases of public health policies, such as the North Karelia Project [11]. This consisted of the implementation of policies aimed at reducing the mortality rate due to coronary diseases in the state of North Karelia (Finland). Mass campaigns were carried out to change the habits of individuals in the population of interest (North Karelia province) and the results were compared with the neighbouring population (Kuopio province). Mortality rates due to coronary disease decreased in both populations, so it was very difficult to measure an actual effect of the intervention. This occurred because the actions carried out in North Karelia also influenced individuals in Kuopio, modifying the causal structure of the "control" region as well. This problem could have been avoided by knowing the social mechanisms involved.

\section{When Mechanisms Speaks about Causality}

On the other hand, evidence of mechanisms may clarify that the results of a clinical trial are causal effects of treatment and not simply the product of correlations between variables. This argument finds support in Russo and Williamson's [12] epistemological thesis of causality: "To establish that A is a cause of $B$ in medicine one normally needs to establish two things. First, that A and B are properly correlated--typically, that A and B are probabilistically dependent, conditional on other known causes of B. Second, that there is a mechanism linking A and B that can explain the difference that A generates in B." They argue that, in the health sciences, to confirm a causal relationship between events (for example, the symptoms of a disease and an infectious agent, or the effect of a drug on a certain physiological function) it is not sufficient to rely on a single type of evidence since each is subject to its own limitations (known as "explanatory monism"). Instead, they argue that there are many types of causes coexisting, most notably mechanistic and probabilistic explanations [12]. Therefore, it would be expected to arrive at a better medical decision in those 
cases where the determination of the causes of a condition, or the observed results of the use of a treatment, is based on both evidence of mechanisms and evidence of correlation.

Failure to consider the underlying mechanisms risks accepting false causality in purely correlational statistical studies. For example, a randomized controlled trial in patients with a blood infection found a decrease in hospital stay in those who were assigned to a treatment consisting of a group of people praying for them at a distance. The authors concluded that there is an association (correlation) between saying prayers to patients and faster recovery, but caution that "there is no known mechanism today that can explain the effect of remote, retroactive intercessory prayer to a group of patients with a blood infection", so it is not correct to speak of a causal relationship [13]. Other examples like this can be found in studies of precognition and homeopathy.

\section{Conclusion}

The mechanistic framework has developed in a very considerable way recently in the philosophy of science, especially in the biological sciences, neurosciences and health sciences. Mechanisms refer to a set of entities and activities that are related in a way that allows for the transition from initial to final conditions. Knowing the mechanisms that underlie a given phenomenon allows for much more than just explaining it. For example, knowing the neurological mechanisms of certain behaviors allows us to generate intervention strategies to improve a cognitive function or alleviate a mental disorder. Also, consideration of the mechanisms can, in conjunction with other forms of evidence, greatly improve the practice of medicine in several different ways by avoiding falling into incorrect assumptions and false causalities. Hence, the knowledge of mechanisms is claimed to be a vital source of reliability in the reflective analysis of the advances of science and their consequences in the resolution of concrete problems in the world.

\section{References}

1. Machamer P, Darden L, Craver C (2000) Thinking about Mechanisms. Philosophy of Science 67(1): 1-25.

2. Craver CF, Tabery J (2019) Mechanisms in Science, the Stanford Encyclopedia of Philosophy (Summer 2019 edn.), Edward N Zalta (Ed.).

3. Craver CF (2007) Explaining the Brain: Mechanisms and the Mosaic Unity of Neuroscience, Oxford Clarendon Press: 272.

4. Sackett DL, Rosenberg WM, Gray JA, Haynes RB, Richardson WS (1996) Evidence based medicine: what it is and what it isn't. BMJ 312(7023): 71-72.

5. Clarke B, Gillies D, Illari P, Russo F, Williamson J (2014) Mechanisms and the evidence hierarchy. Topoi 33(2): 339-360.

6. (2009) NICE, The guidelines manual. National Institute for Health and Clinical Excellence, London.

7. Clarke B, Gillies D, Illari P, Russo F, Williamson J (2013) The evidence that evidence-based medicine omits. Preventive Medicine 57(6): 745747.

8. Bickle J (2019) Multiple Realizability, The Stanford Encyclopedia of Philosophy (Spring 2019 edn.), Edward N Zalta (Ed.).

9. (2011) Food and Drugs Administration. Soliris (Eculizumab), Highlights of prescribing information.

10. Victora CG, Habicht JP, Bryce J (2004) Evidence-based public health: Moving beyond randomized trials. Am J Public Health 94(3): 400-405.

11. Puska P, Vartiaine E, Laatikainen T, Jousilahti P, Paavola M (2009) The North Karelia Project: from North Karelia to national action. National Institute for Health and Welfare.

12. Russo F, Williamson J (2007) Interpreting causality in the health sciences. Int Stud Philos Sci 21(2): 157-170.

13. Leibovici L (2001) Effects of remote, retroactive intercessory prayer on outcomes in patients with bloodstream infection: a randomized controlled trial. Br Med J 323(7327): 1450-1451.

\section{ISSN: 2574-1241}

DOI: $10.26717 /$ BJSTR.2020.25.004163

Ignacio Gimenez. Biomed J Sci \& Tech Res

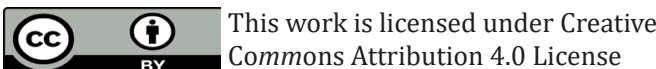

Submission Link: https://biomedres.us/submit-manuscript.php

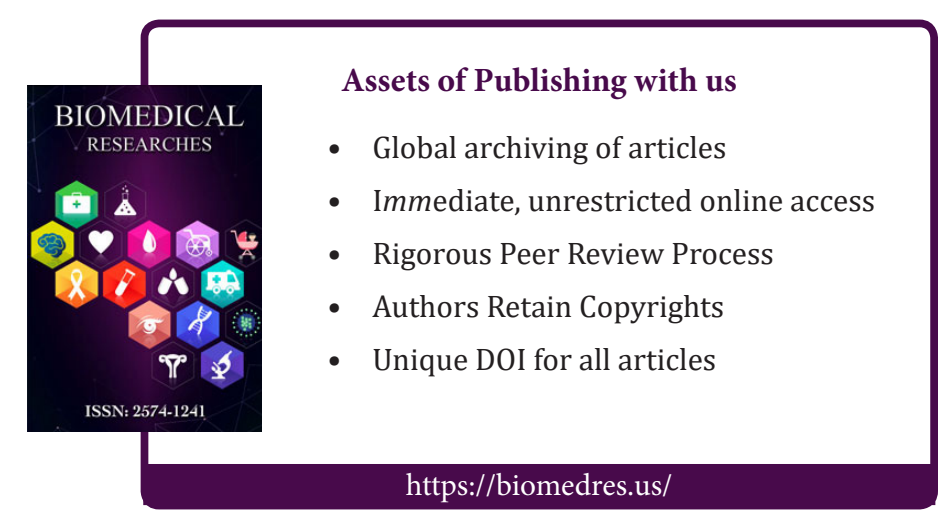

